\title{
Bacteriologically Confirmed Neuromeningeal Tuberculosis at the Hubert Koutoukou Maga National Hospital and University Center in Cotonou: About Two Cases Study and a Review of the Literature
}

\author{
Angèle Azon-Kouanou1, Kouessi Anthelme Agbodande1, Leoubou Roger Samuel Dodo1, \\ Mahoutin Semassa Ghislain Missiho', Roberto Dossou Torès Kouassi Prudencio', \\ Delphin Murhula1, Marius Doukpo', Eugénie Dansou1, Thomas Lokossou², \\ Djimon Marcel Zannou ${ }^{1}$, Fabien Houngbe ${ }^{1}$
}

\begin{abstract}
${ }^{1}$ Internal Medicine Department of CNHU-HKM, Cotonou, Benin
${ }^{2}$ Multi-Purpose Anesthesia and Resuscitation Department of CNHU-HKM, Cotonou, Benin

Email: angele.azonkouanou@gmail.com, agbotem@yahoo.fr, dodo.roger@yahoo.fr, missiho.ghislain@yahoo.fr, prudenciotores@gmail.com, delphinmurhula@gmail.com, doukpom@gmail.com, eugeniedansou1@gmail.com, thomas_comlan@yahoo.fr,djmzannou@yahoo.fr, fab2012jos@yahoo.com
\end{abstract}

How to cite this paper: Azon-Kouanou, A., Agbodande, K.A., Dodo, L.R.S., Missiho, M.S.G., Prudencio, R.D.T.K., Murhula, D., Doukpo, M., Dansou, E., Lokossou, T., Zannou, D.M. and Houngbez, F. (2020) Bacteriologically Confirmed Neuromeningeal Tuberculosis at the Hubert Koutoukou Maga National Hospital and University Center in Cotonou: About Two Cases Study and a Review of the Literature. Open Journal of Internal Medicine, 10, 190-197. https://doi.org/10.4236/ojim.2020.102020

Received: March 31, 2020

Accepted: May 16, 2020

Published: May 19, 2020

\section{Copyright $\odot 2020$ by author(s) and} Scientific Research Publishing Inc. This work is licensed under the Creative Commons Attribution International License (CC BY 4.0). http://creativecommons.org/licenses/by/4.0/ cc) (i) Open Access

\begin{abstract}
Introduction: Tuberculosis remains a major public health problem. The damage to the central nervous system is severe. We reported here two cases. Clinical Case 1: A 37-year-old female patient, naively Human Immunodeficiency Virus1 (HIV1)-positive with antiretroviral (ARV) treatment, hospitalized in the Internal Medicine department for altered consciousness in a febrile context. The clinical examination at admission noted a meningeal syndrome. Cytological analysis of the cerebrospinal fluid (CSF) revealed lymphocytic meningitis with $98 \%$ lymphocytes for 3634 leukocytes. CSF polymerase chain reaction (PCR) detected Mycobacterium tuberculosis DNA. She was put on anti-tuberculosis treatment. The immediate course was favourable, but a secondary worsening of the clinical picture was the cause of his death. Clinical Case 2: A 34-year-old male patient, naively HIV1-positive with ARV treatment. He is hospitalized in the Multi-Purpose Anaesthesia and Resuscitation Department of the CNHU HKM for altered consciousness in a feverish context. The clinical examination noted a meningeal syndrome. The cytological analysis of the CSF noted 184 leukocytes for 99\% lymphocytes. The CSF PCR identified the DNA of Mycobacterium tuberculosis. He was put on anti-tuberculosis treatment. The evolution was marked by his death. Con-
\end{abstract}


clusion: The neuromeningeal localization of tuberculosis is a poor prognosis. Co-infection with HIV remains a potential deadly combination.

\section{Keywords}

Neuromeningeal Tuberculosis, HIV Infection, Death, Cotonou

\section{Introduction}

Tuberculosis (TB) is common worldwide with nine million new cases and two million deaths annually [1]. It is one of the most widespread infectious diseases in the world and a major public health problem; especially in countries with limited resources [2] [3] [4]. In Benin in 2015, the incidence of TB was 60/100,000 inhabitants [5]. The pulmonary form of this condition is the most frequent form of bacillary disease and is a major concern for management services. Among the described extra-pulmonary forms of this condition, tubercular meningoencephalitis accounts for 5\% to $15 \%$ of these locations [6]. This localization of Mycobacterium tuberculosis infection remains a serious form because despite progress in anti-tuberculosis antibiotic treatment and resuscitation, most of time it always involves a life-threatening prognosis [6] [7]. This severity of the condition is secondary to the delay in the diagnosis and management. The most common reasons for late diagnosis are: difficulties in accessing care in resource-limited countries; the presence of mild somatic symptoms wrongly attributed to systemic infection in the prodromal phase of the disease; and misinterpretation of cerebrospinal fluid (CSF) abnormalities wrongly attributing meningitis to a cause due to the difficulty of detecting mycobacterium tuberculosis in the CSF [7] [8] [9]. Diagnosis of certainty was made by standard examinations such as direct examination and culture. To date, the polymerase chain reaction (PCR) technique by Gen Xpert is the only alternative to standard techniques [10]. It allows a rapid diagnosis and is reported as the best diagnostic tool of this location compared to standard examinations according to several authors [7] [10] [11].

The diagnosis of neuromeningeal tuberculosis in our context was based on indirect arguments as well as on standard tests. The present article through these two clinical cases aims to show the important place of Polymerase Chain Reaction (PCR) in the diagnosis. The interest of this work is to make any clinician in charge of a patient presenting neurological symptoms aware of the systematic search for Mycobacterium tuberculosis in the CSF by the Gen Xpert technique in the endemic context of tuberculosis.

\section{Clinical Case 1}

A 37-year-old woman, followed for high blood pressure, was admitted to the Internal Medicine Department of the CNHU-HKM in Cotonou for altered consciousness. The story began a few days before his admission with unusual asthe- 
nia, anorexia, intense headache in a helmet, all in a fever context. The occurrence a few days later of an alteration of consciousness motivates the use of care. She had no notion of vomiting, photophobia, or phonophobia. The general state at the entrance was altered with a WHO performance index of 4 and a systemic inflammatory response syndrome (temperature at $39.5^{\circ} \mathrm{C}$, pulse at 123 beats per minute, respiratory rate at 21 cycles per minute). On physical examination, there were an obnubilation table with a Glasgow score of 11 out of 15 (E4V2M5) and a meningeal syndrome with (stiff neck, presence of a Kerning and Brudzinski Des were without particularity. In front of this painting, a meningoencephalitis was mentioned. A lumbar puncture revealed a clear hypertensive CSF. The CSF examination noted: a predominantly lymphocyte-dominant liquid with $98 \%$ on 3634 leukocytes $/ \mathrm{mm}^{3}$; hypoglycorachia at $0.33 \mathrm{~g} / \mathrm{l}$ for instantaneous venous blood glucose at $0.8 \mathrm{~g} / \mathrm{l}$, and hyperproteinorachy at $1.63 \mathrm{~g} / \mathrm{l}$. The staining of the CSF with Chinese ink in search of Cryptococcus Neoformans was negative. There were no germs in the gram stain, and no soluble antigen. The search for BAAR was negative on direct examination of the CSF, while the DNA of Mycobacterium tuberculosis by the Gen Xpert technique was positive and sensitive to rifampicin. The blood count showed inflammatory anemia (hemoglobin level at $6.2 \mathrm{~g} / \mathrm{dl}$, GMV at 79.4 $\mathrm{fl}$, TGMH at $22.1 \mathrm{pg}$, reticulocytes at $8.7 \mathrm{G} / \mathrm{l}$ ). There is a biological inflammatory syndrome with a high Protein Reactive C of $82 \mathrm{mg} / \mathrm{l}$, the sedimentation rate was accelerated to $76 \mathrm{~mm}$ in the first hour. The blood ionogram and calcium and phosphorus balance were normal. The thick drop and parasitic density were negative, and the chest X-ray was normal. HIV serology was positive for HIV 1 with a TCD4 lymphocyte count of 36 cells/ $\mu \mathrm{L}$. The diagnosis of opportunistic meningoencephalitis type $\mathrm{BK}$ opportunistic disease on retrovirus field of recent discovery not yet initiated with ARV treatment was retained. The patient was placed on the fixed combination Etamubutol-Rifampicin-Isoniazid-Pyrazinamide (ERHZ) 4cp/day and Cotrimoxazole $480 \mathrm{mg} \mathrm{cp}: 2 \mathrm{cp} /$ day by nasogastric tube, and supplemented by ARV treatment (Tenofovir-Lamivudine-Efavirenz). The blood cell transfusion was also carried out. The evolution was initially favourable with a return to a normal consciousness, the amendment of fever, with the disappearance of the meningeal syndrome. The analysis of the CSF after the lumbar puncture control at D4 reveals the presence of 210 lymphocytes $/ \mathrm{mm}^{3}$, or $5.8 \%$ against $98 \%$ initially. On the 20th days of hospitalization, there was a rise in temperature associated with respiratory distress. The blood culture carried out identified multi-resistant escherichia coli. The diagnosis of a nosocomial infection was therefore made, and the patient died in this context.

\section{Clinical Case 2}

A man of 34 years old, with no particular antecedent, was admitted to the general anaesthesia and resuscitation department of the CNHU-HKM in Cotonou for alteration of consciousness. The story began a few days before his admission with an unquantified fever, intense asthenia and anorexia. The onset of vomiting 
a few days later and a progressive alteration of the state of consciousness motivates the use of care. There was an alteration in general condition with a WHO performance index of 4 and a systemic inflammatory response syndrome (temperature at $39^{\circ} \mathrm{C}$; pulse 110). The physical examination noted an altered consciousness with a Glasgow score of 8/15 (E3V1M4); and a stiff neck isolated. In addition, the examination of the other devices was without particularity. In front of this painting, a meningoencephalitis was mentioned. Paraclinical examinations performed showed: Clear cerebrospinal fluid, hypertensive with predominantly lymphocytic predominance (99\% lymphocytes out of 184 leukocytes $/ \mathrm{mm}^{3}$ ) with hypoglycorachia at $0.45 \mathrm{~g} / \mathrm{l}$ and hyperproteinorachia at $2.5 \mathrm{~g} / \mathrm{l}$. The staining of the CSF with Chinese ink in search of Cryptococcus Neoformans was negative. There were no germs in the gram stain, and no soluble antigen. The search for BAAR was negative on direct examination of the CSF, while the DNA of Mycobacterium tuberculosis by the Gen Xpert technique was positive and sensitive to rifampicin. The blood count was normal. The ionogram noted a hyponatremia at $124 \mathrm{meq} / \mathrm{l}$. HIV serology was positive for HIV 1 with a TCD4 lymphocyte count of 18 cells $/ \mu \mathrm{L}$. The diagnosis of opportunistic meningoencephalitis type BK opportunistic disease on recently discovered retrovirus field not yet initiated in complicated ARV treatment of hyponatremia has been retained. The patient was placed on the fixed combination ERHZ 4cp/day and Cotrimoxazole $480 \mathrm{mg}$ cp: $2 \mathrm{cp} /$ day by nasogastric tube with correction of his hyponatremia. The progression under this treatment was unfavorable. Death occurred 5 days after initiation of TB drug therapy in a context of severe impairment of general condition. ARV treatment could not be initiated.

\section{Discussion}

Tuberculosis remains a real public health problem in developing countries today [8] [12]. Central nervous system involvement by Mycobacterium tuberculosis defines neuromeningeal tuberculosis. This disease localization accounts for 5\% to $15 \%$ of extra-pulmonary tuberculosis [6] [12]. Neuromeningeal damage is mainly caused by bloodborne dissemination from a site usually in the lung [7] [12]. It may also be secondary to an endogenous reaction of a granuloma meningeal tuberculosis probably contemporary with the primary infection that had remained latent until then [7]. The time between this latent granuloma and meningitis is very variable and ranges from 6 months to 2 years in adults, rarely more [7]. This mechanism of meningeal damage to the pathology could be one of our patients since these patients did not have any lung damage. It is an affection of the young subject socially and economically active. Most studies conducted on this subject report an average age of 20 to 40 years [8] [10] [12] [13]. The age of our patients, 37 years and 34 cases respectively, confirms this observation. The risk factors recognized in the literature are: poor socio-economic conditions, HIV infection, alcoholism, diabetes, pregnancy, and long-term corticosteroid therapy [8]. Among our patients, HIV infection was the risk factor 
we found. This HIV infection remains a public health problem. Its emergence since the 1980s has led to a significant increase in TB incidence and mortality in many countries, particularly in Africa [14]. As it progresses, it depresses the immune system and promotes rapid reactivation of a still latent meningeal focus or spread of tuberculosis from a primitive focus to the meninges. Our first case had significant immunosuppression with TCD4 lymphocyte levels at 36 cells $/ \mu \mathrm{L}$. It is therefore important to strengthen early detection and management of infected individuals in order to prevent tuberculosis, which is recognized as the leading cause of death in these individuals. Clinically, the disease progresses in several phases. The first phase is the prodromal phase. This prodromal phase of CNS tuberculosis is characterized by moderate fever, sweating, loss of appetite, tendency to daytime drowsiness, headache, irritability and, more rarely, mood and behaviour disorders without constituting a true psychiatric picture [15] [16]. These are therefore non-specific symptoms, making early diagnosis difficult. It is only during the state phase that the clinical picture becomes clearer, with the appearance of confounding symptoms and a neuro-meningeal syndrome, with an alteration of the general state. Our patients were seen in the state phase since altered consciousness and syndrome meningitis were present at admission. On the paraclinical level, the biochemical examination of the CSF generally notes a proteinorachy that is greater than $1 \mathrm{~g} / \mathrm{l}$, which, among meningitis with clear liquid, is a first element of differentiation of viral meningitis [7]. This important meningeal exudate was observed in our patients with respective levels of $1.63 \mathrm{~g} / \mathrm{l}$ and $2.5 \mathrm{~g} / \mathrm{l}$. The glycorachia is constantly lowered and remains so for several weeks. This is a crucial biochemical data for the etiological diagnosis of clear liquid meningitis, justifying the determination of blood glucose levels before any lumbar puncture, so that hyperglycemia does not mask early hypoglycorachia [7]. This hypoglycorachia was also observed in our patients. Hypochlorurorachy has been observed, and this is an important sign of orientation [7]. It is largely the consequence of the hyponatremia that often accompanies tuberculous meningitis [7]. This hyponatremia was noted in the second patient. It must be detected and treated because it could increase the risk of death. In CSF cytology, the cellular reaction is made up of lymphocytes, 200 to $300 \mathrm{~mm}$, sometimes greater than 1000 with a high percentage of polynuclear cells gradually replaced by lymphocytes [7]. Our patients had a highly lymphocytic cytology which led to the diagnosis. The exclusive presence of polynuclear cells is possible in the very first days, before lymphocytosis develops [7].

This means that in the case of meningitis with predominantly neutrophilic cytology, the diagnosis of tuberculosis should not be formally ruled out in favour of purulent meningitis, especially in an HIV-infected person. It must always be kept in mind. Very close monitoring of the patient must be carried out and the unfavourable evolution of the clinical picture under well-conducted non-specific antibiotics must lead to the diagnosis being questioned and another lumbar puncture with cytological examination discussed. In short, it is always necessary 
to look for Mycobacterium tuberculosis in the CSF during meningitis, regardless of its cellular prevalence, since we are in an endemic tuberculosis context. On bacteriological examination by the CSF, the detection of Mycobacterium tuberculosis makes it possible to make a diagnosis with certainty. In the past, it used standard examinations such as the direct examination. Identification of Mycobacterium tuberculosis in the CSF on direct examination after centrifugation varies between $7 \%$ and $40 \%$ depending on the series [7] [10]. It has a low germ detection capability. This was observed by Dollo, I. et al. [10] and Miliani, M. et al. [12] who reported a low germ detection rate in the CSF with rates of $1.9 \%$ and $0 \%$ respectively. In our series, the direct examination for germ was also negative. The culture of the cerebrospinal fluid on specific medium allowed the diagnosis to be made with a delay of 3 to 4 weeks. This is relatively long for such a serious pathology. This examination was not done in our patients. To date, the Polymerase Chain Reaction (PCR) technique is the only alternative to standard techniques [7] [10]. It is currently described as having a higher germ detection capacity than culture on specific medium. This is shown by Dollo, I. et al. [10] who reported that PCR and culture on specific medium allowed the germ to be detected at frequencies of $92 \%$ and $84 \%$ respectively. De Seze, J. [11] made the same observation with respective frequencies of $45 \%$ and $18 \%$.

There are several advantages to this review. It allows a rapid diagnosis, and also identifies problems of resistance to major anti-tuberculosis drugs in the area of multidrug-resistant tuberculosis. It is important to stress that its negativity does not totally exclude diagnosis. Neuromeningeal tuberculosis is a serious pathology with a high risk of mortality. It is responsible for death and serious neurological sequelae in more than $50 \%$ of cases despite well-conducted antituberculosis treatment [8] [10] [17]. In our case, the evolution was unfavorable with the occurrence of death in both patients. According to the British Medical Research Council [18], clinical criteria for the severity of tubercular meningitis have been defined, and three stages have been differentiated: the first corresponds to the prodromal phase where there are no confounding or neurological symptoms, with mortality below $10 \%$, while this increases to $37 \%$ and up to $87 \%$ in stages 2 and 3 which correspond to the state phase. This high mortality and the frequency of neurological sequelae, show the importance of early diagnosis, especially during the prodromal phase. The fact that our patients are seen in the state phase could then explain the occurrence of their death.

\section{Conclusion}

Neuromeningeal tuberculosis is a serious form of TB, usually affecting the immunocompromised individual. Its prognosis is correlated with the stage of the disease at which the diagnosis was made. Despite advances in antibiotic treatment and resuscitation, the risk of mortality is very high when diagnosed in the status phase. In order to reduce the mortality associated with the condition, it is therefore important to strengthen the early detection and management of 
HIV-infected persons and also make an early diagnosis of the disease in the prodromal phase.

\section{Authors' Contributions}

All the authors contributed to the conduct of this work (literature review and writing of the manuscript).

\section{Conflicts of Interest}

The authors declare no conflicts of interest regarding the publication of this paper.

\section{References}

[1] Mazza-Stalder, J., Nicod, L. and Janssens, J.P. (2012) Extrapulmonary Tuberculosis. Respiratory Disease Review, 29, 566-578. https://doi.org/10.1016/j.rmr.2011.05.021

[2] Dagnra, A.Y., Adjoh, K., Tchaptchet, H.S., Patassi A.A., Sadzo, H.D., Awokou, F., et al. (2011) Prevalence of HIV-Tuberculosis Co-Infection and Impact of HIV Infection on the Evolution of Pulmonary Tuberculosis in Togo. Bulletin de la Société de Pathologie Exotique, 104, 342-346. https://doi.org/10.1007/s13149-010-0079-3

[3] Cherif, J., Ben Salah, N., Toujani S., Ouahchi, Y., Zakhama, H., Louzir, B., Mehiri-Ben, Rhouma, N. and Beji, M. (2014) Epidemiology of Tuberculosis. Revue de Pneumologie Clinique, 71, 67-72. https://doi.org/10.1016/j.pneumo.2014.04.002

[4] Horo, K., Touré, K., Brou-Gode, V.-C., AhuB, J.M.I., Kouassi, B.A., Gnazé, A.Z., et al. (2012) Tuberculosis in the Elderly: Epidemiology and Becoming Outpatients in Abidjan. Revue dépidémiologie et de Santé Publique, 60, 484-488. https://doi.org/10.1016/j.respe.2012.02.009

[5] Word Health Organisation (2015) Incidence of Tuberculosis per 100,000 People. Global Tuberculosis Report. https://data.worldbank.org/indicator/SH.TBS.INCD?locations=BJ

[6] Katti, M.K. (2004) Pathogenesis, Diagnosis, Treatment, and Outcome Aspects of Cerebral Tuberculosis. Medical Science Monitor, 10, 215-229.

[7] Bazin, C. (2004) Tuberculosis of the Central Nervous System. EMC: Neurologie, 1, 169-192.

[8] Khalid, M., Tarik, B. and Abdelhadi, R. (2017) Tuberculous Meningoencephalitis Revealed by Psychiatric Disorders: About A Case. Pan African Medical Journal, 27, 206.

[9] Anderson, N.E., Somaratne, J., Mason, D.F., Holland, D. and Thomas, M.G. (2010) A Review of Tuberculous Meningitis at Auckland City Hospital, New Zealand. Journal of Clinical Neuroscience, 17, 1018-1022. https://doi.org/10.1016/j.jocn.2010.01.007

[10] Dollo, I., Fane, E.M., Es-Sebbani, M., Chakib, A., Sodgi, M., Ouladlahsen, A. et al. (2017) Confirmed Tuberculous Meningitis: About 52 Cases. Pratique Neurologique $F M C, 8,168-173$. https://doi.org/10.1016/j.praneu.2017.06.011

[11] De Seze, J., Deligne, L., Defebvre L., Ferriby, D., Charpentier P., Stojkovic, T., et al. (2004) Neuromeningeal Tuberculosis: Contribution of the Gene Amplification Method to Diagnosis. Revista de Neurología (Paris), 160, 413-418.

https://doi.org/10.1016/S0035-3787(04)70922-4 
[12] Miliani, M. (2016) Neuromeningeal Tuberculosis. Retrospective Study of a Series of 16 Cases with Literature Review. M.D. Thesis, Université SIDI Mohammed Ben Abdellah (Faculty of Medicine and Pharmacy), Maroc. N¹66/16. 16 p.

[13] Raveloson, N.E., Rakotoarivony, S.T., Rasamimanana, N.G., Raharimanana, N.R., Andrianjatovo, J.J. and Sztark, F. (2009) About a Case of Meningitis Tuberculosis of Deceptive Form. Journal of Anesthesia-Resuscitation and Emergency Medicine, 1, 11-13.

[14] Lot, F., Pinget, R., Cazein, F., Pillonel, J., Leclers, M., Haguy, H., et al. (2009) Frequency and Risk Factors of Inaugural AIDS Tuberculosis in France. Bulletin Épidémiologique Hebdomadaire, 13, 289-293.

[15] Cherian, A. and Thomas, S.V. (2011) Central Nervous System Tuberculosis. African Health Sciences, 11, 116-127.

[16] Thwaites, G.E. and Hien, T.T. (2005) Tuberculous Meningitis: Many Questions, too Few Answers. The Lancet Neurology, 4, 160-170. https://doi.org/10.1016/S1474-4422(05)70019-3

[17] Thwaites, G.E., Bang, N.D., Dung, N.H., Quy, H.T., Oanh, T.T., Thoa, N.T.C., et al. (2004) Dexamethasone for the Treatment of Tuberculous Meningitis in Adolescents and Adults. The New England Journal of Medicine, 351, 1741-1751. https://doi.org/10.1056/NEJMoa040573

[18] The British Medical Research Council (2016) Clinical Stages for Tuberculous Meningitis. http://www.meducator3.net/.../british-medical-research-council-bmrc 